\title{
Position-resolved Surface Characterization and Nanofabrication Using an Optical Microscope Combined with a Nanopipette/Quartz Tuning Fork Atomic Force Microscope
}

\author{
Sangmin An ${ }^{1}$, Baekman Sung ${ }^{1}$, Haneol Noh ${ }^{1}$, Corey Stambaugh ${ }^{2}$, Soyoung Kwon ${ }^{1}$, Kunyoung Lee ${ }^{1}$, \\ Bongsu Kim ${ }^{1}$, Qhwan Kim ${ }^{1}$, Wonho Jhe $\mathrm{Jn}^{1, *}$
}

(Received 07 October; accepted 12 December 2013; published online 13 January 2014)

\begin{abstract}
In this work, we introduce position-resolved surface characterization and nanofabrication using an optical microscope $(\mathrm{OM})$ combined with a nanopipette-based quartz tuning fork atomic force microscope (nanopipette/QTF-AFM) system. This system is used to accurately determine substrate position and nanoscale phenomena under ambient conditions. Solutions consisting of $5 \mathrm{~nm} \mathrm{Au} \mathrm{nanoparticles,} \mathrm{nanowires,} \mathrm{and}$ polydimethylsiloxane (PDMS) are deposited onto the substrate through the nano/microaperture of a pulled pipette. Nano/microscale patterning is performed using a nanopipette/QTF-AFM, while position is resolved by monitoring the substrate with a custom OM. With this tool, one can perform surface characterization (force spectroscopy/microscopy) using the quartz tuning fork (QTF) sensor. Nanofabrication is achieved by accurately positioning target materials on the surface, and on-demand delivery and patterning of various solutions for molecular architecture.
\end{abstract}

Keywords: Surface characterization; Nanopipette; QTF-AFM; Optical microscope

Citation: Sangmin An, Baekman Sung, Haneol Noh, Corey Stambaugh, Soyoung Kwon, Kunyoung Lee, Bongsu Kim, Qhwan Kim and Wonho Jhe, "Position-resolved Surface Characterization and Nanofabrication Using an Optical Microscope Combined with a Nanopipette/Quartz Tuning Fork Atomic Force Microscope", Nano-Micro Lett. 6(1), 70-79 (2014). http://dx.doi.org/10.5101/nml.v6i1.p70-79

\section{Introduction}

Nanofabrication is an important research field in nanoscale science and technology, and is useful for various applications, such as scientific and technical breakthroughs, openings of new industrial markets, and influence of human life [1]. There are several common nanofabrication methods, including nanoimprinting $[2,3]$, photolithography, E-beam lithography [4,5], and physical/chemical vapor deposition (PVD/CVD). Scanning probe microscope (SPM)-based nanofabrication is capable of yielding nanoscale resolution of fabricated objects [6-8].
There are several pipette (aperture)-assisted micro/nanofabrication methods used for material delivery among the SPM-based nanofabrication techniques. These include controlled delivery with nanopipettes $[9,10]$ and hollow atomic force microscopy (AFM) pyramids in which a probe is milled by focused ion beam lithography with high resolution and throughput [1113]. Integrated carbon pipes with sub-micron diameters are also useful for investigating cell biology and functional patterned microfluidics. These pipes possess small diameters ranging from $20 \mathrm{~nm}$ to microns and have stiffer tips [14]. Carbon-based nanoprobes can also be used as carbon nanopipettes for nanoprint-

\footnotetext{
${ }^{1}$ Center for Nano-Liquid, Department of Physics and Astronomy, Seoul National University, Daehak-dong, Gwanak-gu, Seoul 151-747, South Korea

${ }^{2}$ National Institute of Standards and Technology, MD 20899, USA

*Corresponding author. E-mail: whjhe@snu.ac.kr
} 
ing and drop dispensing [15]. Moreover, micropipettes and micromanipulators are used to place micron size beads at designated positions for microfluidic applications $[16,17]$. The nanopipette combined with a tuning fork quartz crystal resonator has been used for nanopatterning of $200 \sim 300 \mathrm{~nm} \mathrm{Cu}$ dots in a liquid environment [18].

The shapes of nanofabricated structures are normally confirmed after the fabrication process using scanning electron microscopy (SEM), transmission electron microscopy (TEM), AFM, etc. Although the objective lens is located above the AFM header in order to select the scanning region of interest, nanofabrication near the tip is difficult to observe in situ because the upper side of the cantilever holding the objective lens obscures the viewing area. To address this issue, several cantilever techniques integrated into an optical microscope (OM) have been introduced for in situ definition, such as cantilever glass micropipettes with atomic control of chrome etching [19] and FluidFM in liquids [20].

Here, we show position-resolved surface characterization and nanofabrication using an optical microscope $(\mathrm{OM})$ combined with a nanopipette/quartz tuning fork atomic force microscope (nanopipette/QTF-AFM) system, which accurately determines substrate position and nanoscale phenomena via atomic force measurements under ambient conditions. Liquid-delivered nanolithography through the nanoscale aperture of a pulled nanopipette attached to the QTF-AFM sensor head is captured using OM images with surface characterization in situ in the desired region. A liquid solution filled nanopipette is attached to the edge of one prong of the quartz tuning fork (QTF) using a commercial epoxy and acts as the tip of the force sensor. This tip is can be reused over 50 times with acetone treatment. The tip approaches within $10 \mathrm{~nm}$ of the substrate to form a nanoscale object by ejecting solution while observing extrusion phenomena in situ using OM. In addition, polydimethylsiloxane (PDMS) is directly patterned and nanowires are aligned as connectors between two pre-coated regions. With this technique, one can accurately position nanostructures and observe the nanofabrication process. Moreover, various liquid solutions can be used to fabricate nanoscale objects on-demand. In addition, the motion of the system can be recorded from the electrical output signal (amplitude and phase) of the QTF sensor using the well-established theory of viscoelasticity for any target objects $[21,22]$. This theory is based on a damped harmonic oscillator model taking into account the effects of elasticity $\left(k_{\text {int }}\right)$ and viscosity $\left(b_{\text {int }}\right)$ of the interacting object. In this experiment, we use the amplitude signal to accurately define the position of the pipette apex from the surface without interpreting the viscoelastic behavior of the fabricated objects.

\section{Experimental}

We demonstrated position-resolved surface characterization and liquid-delivered nanofabrication using various solutions composed of nanoparticles and dye molecules. In situ observation of surface phenomena was made using from a custom-built OM incorporated into the nanopipette/QTF-AFM.

\section{OM combined with Nanopipette/QTF-AFM system}

Figure 1(a) shows a schematic of the proposed OMcombined QTF-AFM system. The nanopipette-based QTF-AFM system is located above the objective lens of the OM as shown. The magnified region of interest is monitored with a $z$-axis high resolution $(0.25 \mu \mathrm{m}) \mathrm{OM}$, consisting of an objective lens $(\times 20, \times 40, \times 60, \times 100)$ and a charge-coupled device $(\mathrm{CCD})$ camera. Images were recorded during the experiment with a clear image of the piptette apex. The pencil-shaped nanopipette ( $\sim 100 \mathrm{~nm}$ aperture) is attached to one side of the QTF prong above the OM. The pulled nanopipette was fabricated using a commercial puller (P-2000, Sutter Instrument Co.), where the aperture diameter was varied by changing the pulling parameters of the machine. The solution is filled up to the apex of the pulled pipette by capillary forces between the inserted filament and the inner wall of the pipette. After pulling, the liquid solution is filled inside the glass pipette via the inserted filament, which is a pre-installed quartz rod (diameter of $\sim 150 \mu \mathrm{m}$ ) inside the wall of the bare pipette wall. This rod is required in order to fill liquid up to the apex of the pulled pipette. Note that the size of the filament, which is small compared to the pipette size (outer diameter of $1 \mathrm{~mm}$, inner diameter of $0.7 \mathrm{~mm}$ ), is reduced at the same rate as the pipette diameter during the pulling process. Moreover, the cross section of the aperture has a planar morphology as observed in SEM images. Although the aperture shape is slightly twisted by the filament during pulling, the shape does not critically affect the experiment. When the opposite side of the apex of the pulled pipette is inserted in the liquid solution, the solution climbs up to the apex via the capillary forces between the inner wall of the pipette and the outer wall of the filament and starts accumulating at the apex. The liquid-filled pipette is attached to one prong of the QTF to sustain a narrow distance between the tip and the glass substrate of less than $10 \mathrm{~nm}$. It is important to position the pipette perpendicular to the substrate. A tilted contact area can affect the response of the QTF sensor through the relatively large contact area (outer diameter of $\sim 180 \mathrm{~nm}$ ) and $10 \mathrm{~nm}$ distance from the surface. Thus, the pulled pipette (total length $\sim 5 \mathrm{~cm}$, length from starting position of the taper to the apex $\sim 0.5 \mathrm{~mm}$ ) is securely mounted on the pipette 

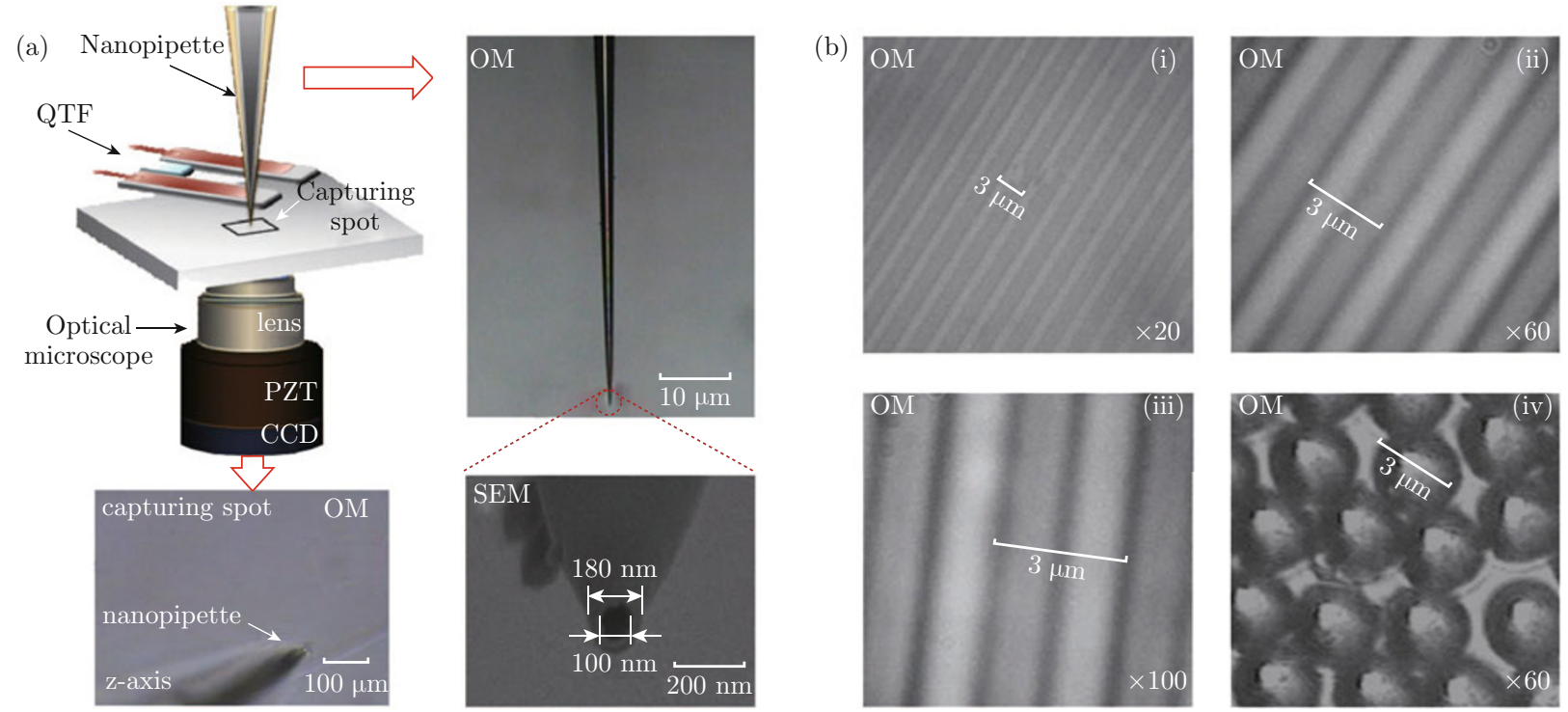

Fig. 1 Proposed OM combined QTF-AFM system. (a) Schematic of the system. The nanopipette/QTF-AFM system is located above the objective lens of the OM. The pencil-shaped nanopipette (aperture of $\sim 100 \mathrm{~nm}$, outer diameter of $\sim 180$ $\mathrm{nm}$ ) is attached to one side of the QTF prong above the OM. A magnified CCD image of the $z$-axis defines the apex of the nanopipette tip. (b) Surface images of a $3 \mu \mathrm{m}$ grating (objective lens of (i) $\times 20$, (ii) $\times 60$, (iii) $\times 100)$ and $3( \pm 0.1) \mu \mathrm{m}$ polystyrene spheres $($ (iv) $\times 60)$ for standardization of the measurements.

mounting stage above the substrate. The angle is checked using the $x$ - and $y$-axis of the CCD cameras. Because the pipette is tightly secured with a screw on the manipulator and the detection system, the inertia of lead wires ( $E$ field) and tubing (air pumping), located $\sim 5 \mathrm{~cm}$ from the apex and surface, have no effect on the QTF sensor resonance, the phase signals, and subsequent measurements within the maximum movement of the tip (each $x$ -,$y$-, $z$-axis: $\sim 70 \mu \mathrm{m}$ ). As a result, an initially detected QTF reference signal is sustained during the experiment. Figure 1(b) shows surface images (capturing spot) of a $3 \mu \mathrm{m}$ grating (objective lens of (i) $\times 20$, (ii) $\times 60$, (iii) $\times 100)$ and $3( \pm 0.1) \mu \mathrm{m}$ polystyrene spheres $((\mathrm{iv}) \times 60)$ for standardization of the measurements of experiment results. Since both temperature and humidity fluctuations influence the results of the proposed nanofabrication system, we designed a double chamber setup to maintain constant temperature and relative humidity within the experimental requirements. Variations in humidity lead to changes in QTF resonance frequency. The temperature variation can affect the whole system due to thermal instability from slight movements. Thus, a double chamber (outer: acryl, inner, metal) and two kinds of anti-vibration systems (sponge and bench-top) were used to maintain temperature $\left(23 \pm 0.2^{\circ} \mathrm{C}\right)$ and relative humidity $(45.2 \pm$ $0.3 \%$ ) within the required control range (thermal drift of $\sim 0.4 \mathrm{pm} / \mathrm{s}$ ) and to form a well-shaped nanoscale liquid bridge channel between the nanopipette tip and the substrate. Note that the size of the pattern depends on the aperture diameter of the pulled pipette, the relative humidity, the drawing speed, and so on. In particular, the aperture diameter is a critical factor for pattern size. As the aperture size of the pipette is increased, the patterning size increases within the aperture boundaries. At high humidity, the pattern size increases since the evaporation rate of the liquid ejection is low under such conditions. $E$-field-induced liquidejection is also difficult to achieve at a low relative humidity of under $15 \%$, because the proposed system uses the naturally confined nanoscale water-meniscus formed between the rim of the pipette apex and the substrate. The volume of the nanoscale confined water is small and not adequate to eject liquid onto the substrate at low bias potential. Also drawing speed affects pattern size in that resolution increases with speed.

\section{QTF sensor}

A non-contact, small modulation $(<1 \mathrm{~nm})$ shear mode QTF-AFM system can be used to control distance and analyze the mechanical properties of fabricated nanostructures [23]. In particular, a shear mode allows the detection of a shear force from the nanoscale liquid meniscus formed between the apex of the nanopipette and the surface [24]. The small force $(<1 \mathrm{nN})$ associated with the mechanical interaction is detected using a highly sensitive QTF sensor and interpreted within the framework of a damped harmonic oscillator model $[25,26]$. The resonance frequency of the QTF was $\sim 32 \mathrm{kHz}$. The oscillation amplitude was $\sim 0.5 \mathrm{~nm}$. As the tip approaching the sample, the resonance frequency of the QTF changes through a perturbation of the nanoscale liquid meniscus. Using the equation for damped harmonic motion, the interaction force can be interpreted as an effective elasticity $\left(k_{\text {int }}\right)$ and viscosity 


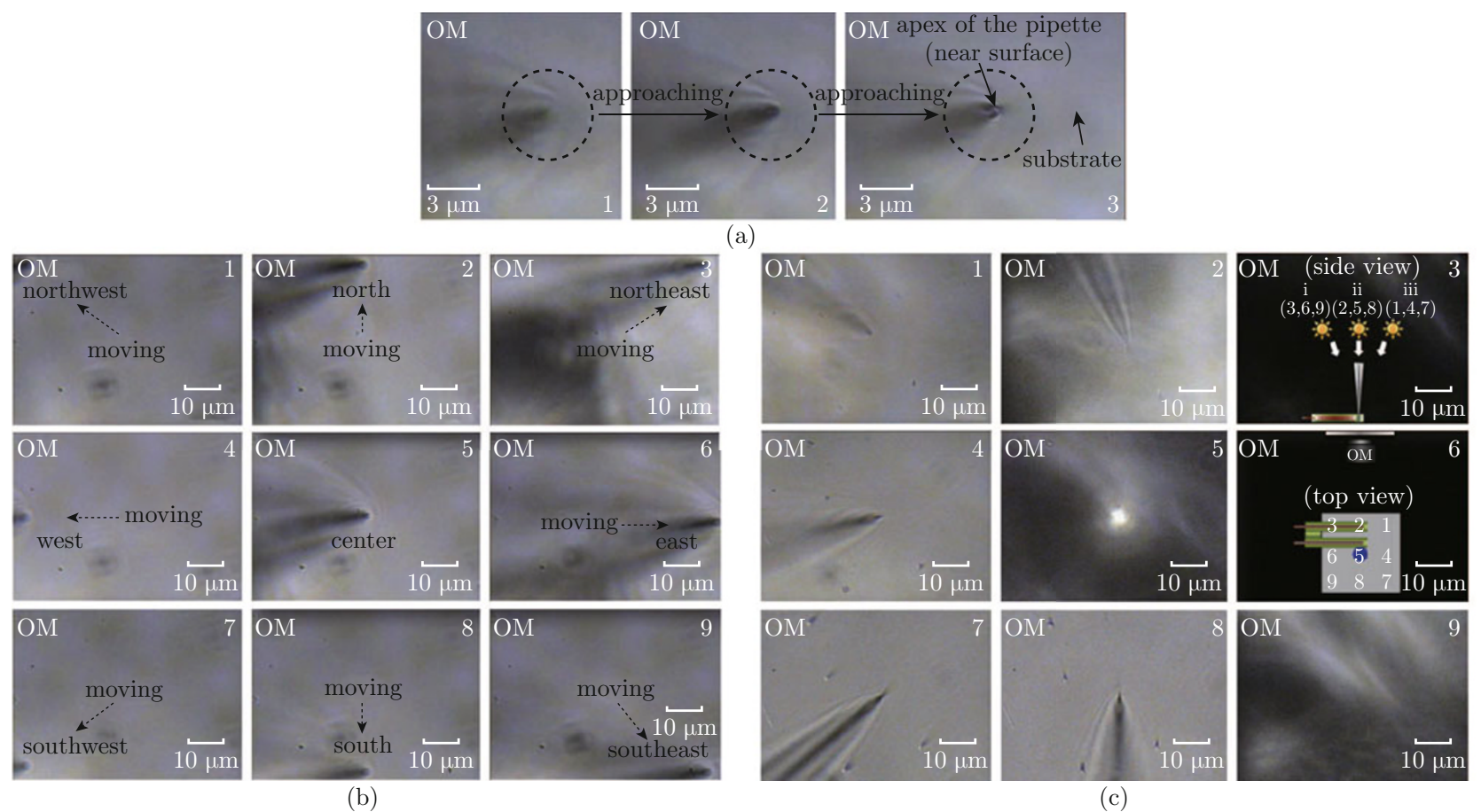

Fig. 2 High definition images of the nanopipette apex acquired with an OM. (a) Images ( $z$-axis) of the pulled nanopipette tip; (b) Lateral movement ( $x$-, $y$-axis) of the nanopipette tip controlled by a commercial PZT transducer; (c) Directional light illumination dependence of the system. The lighting angles of c1, c2, c3, and b1, b3 yield clear images of the nanopipette apex, while shadow images do not clearly distinguish between the nanopipette and the substrate, as in case a1, a2, and a3.

$\left(b_{\text {int }}\right)[27]$. With this theoretical tool, one can study the mechanical properties of nanoscale objects, such as a nanoscale water meniscus.

\section{Definition of the apex of a nanopipette using an $\mathrm{OM}$}

The positioning of a pipette tip on a substrate is important for achieving stable solution-delivered nanofabrication and force detection. However, OM images of the pipette apex near the substrate reveal a tilted shape due to illumination above the region of interest at a certain tilt angle from space limitations. To address this issue, we define the capturing images of the apex of the nanopipette near the surface using an OM with a $\times 60$ objective lens on the $z$-axis. Figure 2(a) shows in situ OM images of the nanopipette tip on the surface. While the incident spot of the tip apex is observed, on-demand surface characterization and nanofabrication are only performed with definition near the contact position. Figure 2(b) shows the lateral movement ( $x$ - and $y$-axis) of the nanopipette tip, which is controlled by a commercial piezoelectric transducer (PZT). The location of the apex of the pipette is clearly defined for all directions (northwest-1, north-2, northeast-3, west-4, center5 , east-6, southwest-7, south-8, and southeast-9). Figure 2(c) shows the changes in images with respect to the direction of light illumination needed to ensure that the pipette is perpendicular to the surface. A white light emitting diode (LED) (THORLABS, LEWWE-15) is used for illumination and to minimize thermal drift in the system. Images 3 and 6 of Fig. 2(c) are side and top view schematics, respectively, of the lighting angle on the system that results in the tilted shadow images of the nanopipette apex. For part i of image 3, which correspond to OM images $3,6,9$, the pipette apex does not obscure the QTF sensor, manipulators, pipette mounting, and so on. However, for part ii $(2,5$, $8)$ and iii $(1,4,7)$ of Fig. 2(c), the apex of the pipette can be clearly observed. These lighting angles (part ii, iii) are frequently used in further experiments except for images using center illumination (5 of part ii). Interestingly, image 5 of Fig. 2(c) (center) shows an intense spot of light due to light focusing from the inner wall of the pipette. This phenomenon can be exploited for nanophotonic applications, such as a near field scanning optical microscope for analysis of mechanical properties or for local heating using highly focused photons.

\section{Results and discussion}

We present surface characterization and in situ fluid/nanofabrication using this nanopipette/QTFAFM system combined with an OM. Electric ( $E$ fieldinduced low volume liquid ejection, material transport, and nanoscale patterning on the glass substrate were investigated using various material solutions, such as nanoparticles, dye molecules, Si nanowire, and PDMS (Dow Corning, Sylgard 184 elastromer kit). Various 
solutions are used for fluid and micro/nanofabrication. The dye molecule solution (rhodamine 6G), which emits green fluorescence when excited by a $488 \mathrm{~nm}$ laser, is using in fluid experiments due to the clear observation of fluid phenomena at the surface. A commercial 5 $\mathrm{nm} \mathrm{Au} \mathrm{nanoparticle} \mathrm{solution}\left(5 \pm 0.2 \mathrm{~nm}, \mathrm{H}_{2} \mathrm{O}\right.$ buffer, $0.01 \%$ concentration, BBInternational Co.) is used for nanofabrication. A low density $\mathrm{Si}$ nanowire solution (50 $\pm 2 \mathrm{~nm}$ diameter, $2 \pm 0.5 \mu \mathrm{m}$ length, $\mathrm{H}_{2} \mathrm{O}$ buffer, $\sim 0.002 \%$ concentration) and PDMS are used for in situ alignment experiments and microfabrication. Dye molecules, Au nanoparticles, and a Si nanowire solution are used with an $E$ field-induced liquid ejection method and a distance regulated AFM system. An air pumping method is used for the PDMS solution through a $3 \mu \mathrm{m}$ aperture of the pulled pipette due to its relatively high viscosity. Note that the electro-osmosis force is used for the dye molecule solution due to the direct contact of liquid onto the electrode. An electrostatic force is only used for liquid ejection for nanofabrication of $\mathrm{Au}$ nanoparticles and Si nanowire solutions.

\section{Surface characterization}

In atomic force microscopy, target regions of scanned images are generally selected at random positions using a CCD camera with a low magnification object lens. Thus, it is difficult to image contact phenomena at the spot of the apex. In order to enable nanoscale in situ surface characterization on a desired spot, a noncontact, shear-mode scanning of frequency modulation ADPLL-based QTF-AFM [28] was performed. With the proposed high resolution OM, the nanopipette tip, which is the same tip used in nanofabrication, is moved on demand to the scan area with a clear image of the apex. Figure 3 shows surface characterization using the nanopipette/QTF-AFM system combined with an OM. The nanopipette tip material, diameter, and dithering amplitude are quartz, $\sim 100 \mathrm{~nm}$, and $\sim 5 \mathrm{~nm}$, respectively. OM and scanned AFM images of the selected region of 3 layered mica surfaces were captured simultaneously in situ and shown in Fig. 3(a). The scanning area and speed are $800 \times 800 \mathrm{~nm}^{2}$ and $0.25 \mathrm{~Hz}$. Distance control was achieved by implementing a digital feedback algorithm embedded on a digital signal processor chip. The positions of the tip $(x-, y$-, $z$-axis $)$ were controlled by changing the output of a 16 bit digital-toanalog converter (NI 6703, National Instruments, Co.). A different 16 bit analog-to-digital converter (NI 6034E, National Instruments Co.) was used to obtain the output signals.

Due to the high stiffness of the QTF, scanning and force spectroscopy of fabricated nanoscale objects are achieved in the non-contact mode operation of the QTF-based AFM with no jump-to-contact. This mode was used to protect the pipette-tip apex from contact damage and allowing repetitive and reliable use with
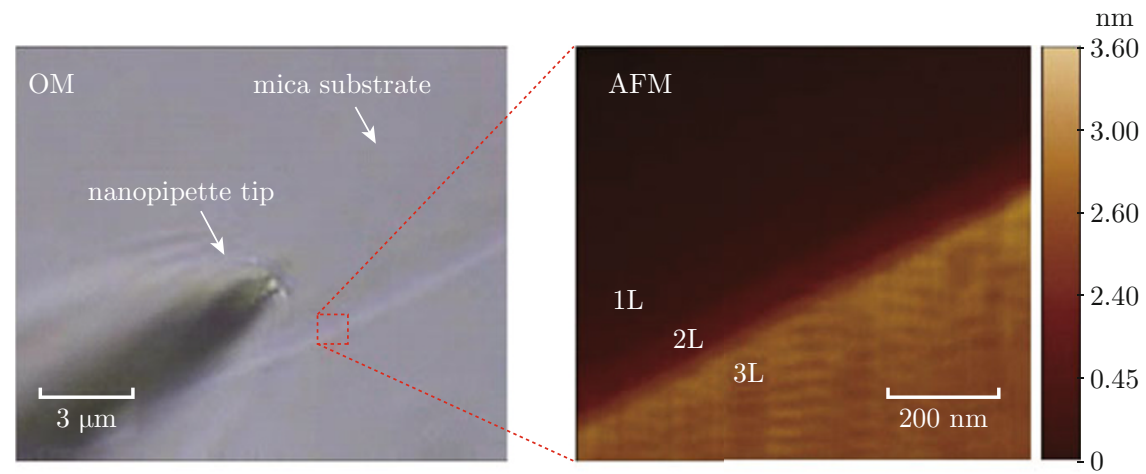

(a)
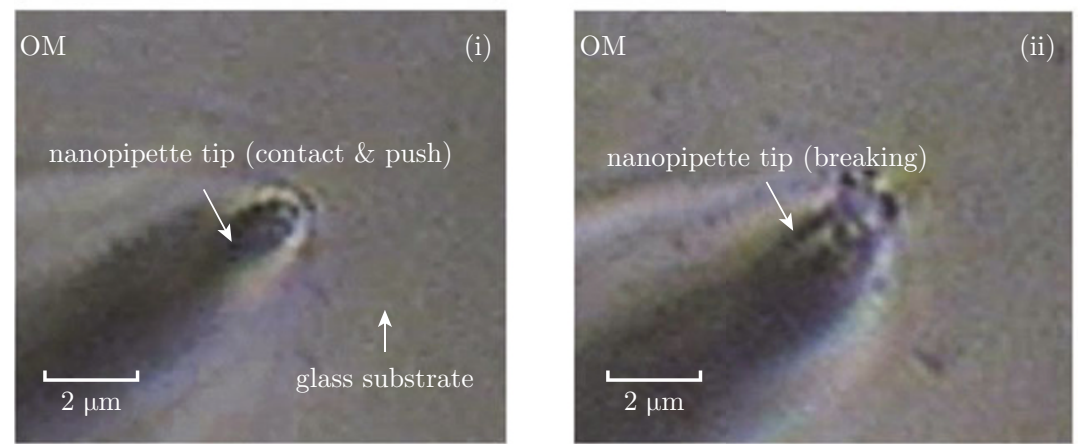

(b)

Fig. 3 Surface characterizations using a nanopipette/QTF-AFM system combined with an OM. (an OM and scanned AFM images for a selected region of three layered mica surfaces. The scanning area and speed are $800 \times 800 \mathrm{~nm}^{2}$ and $0.25 \mathrm{~Hz}$; (b) Images of the contact and breaking of the nanopipette tip as the tip continuously approaches the mica surface. 
sustaining the distance between the tip and the substrate. In the nanofabrication process, small oscillation amplitude $(\mathrm{A} \sim 0.5 \mathrm{~nm}$ ) of the pipette tip attached to the QTF sensor was used for force microscopy due to the minimal amplitude dependence on nanoscale objects. This data is not shown here for clarity. On the other hand, scanning is performed with a large tip oscillation $(\mathrm{A} \sim 20 \mathrm{~nm}$ ) for clearing images of target objects. In the force spectroscopy experiments, the mechanical response in the contact region is important. Figure 3 (b) shows images of the contact and breaking of the nanopipette tip as it continuously approaches the mica surface. The signal from the QTF sensor makes it difficult to define surface phenomena due to a very low quality factor $(\sim 50)$, while the OM yields information on the contact and breaking of the tip.

\section{Fluid and nanofabrications}

Using this tool, fluid and nanobrication are performed as shown in Fig. 4. Figure 4(a) shows a schematic of the fluid and nanofabrication experiments where electrodes are located on or under (corresponding to fluid nanofabrication experiments) a glass substrate (Pyrex@) borosilicate glass, $200 \mu \mathrm{m}$ thickness, $1.5 \mathrm{~cm}$ $\times 1.5 \mathrm{~cm}$ area). The glass substrate is coated using $\mathrm{Au}$ sputtering deposition with $\sim 20 \mathrm{~nm}$ thickness, which is thin enough to avoid obscuring phenomena on the substrate ( $<$ skin depth). The experimental procedure consisted of 3 steps: i) approaching with the application of an $E$ field, ii) liquid ejection, and iii) drawing or retraction. After the tip approaches the surface, capillaryassisted nanoscale liquid ejection and nanofabrication

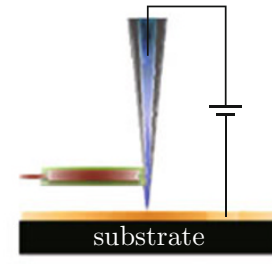

(1) Fluid

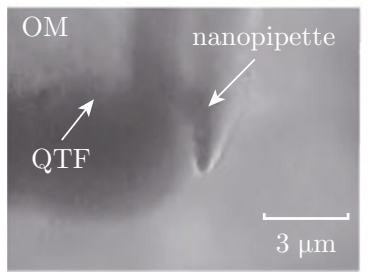

(1) Fluid

(b) are performed with a distance controllable QTF-AFM system $(<10 \mathrm{~nm})$ under the application of an $E$ field [29]. The presence of liquid ejection is confirmed by the sudden change in QTF sensor signals (amplitude and phase) and in situ captured OM images. The flipping of the $\mathrm{Au}$ electrode changes the surface properties, including wetting characteristics and conducting versus insulating properties. We measured the contact angle for each case ( $\mathrm{Au}$ on glass substrate of $\sim 70^{\circ}$, glass of $\sim 20^{\circ}$ ). Liquid ejection was successively performed for each case by applying different bias voltages. For the glass (where the electrode was located under the glass for nanofabrication), a voltage of $\sim 100 \mathrm{~V}$ should be applied instead of $\sim 10 \mathrm{~V}$ (as for Au on glass used for fluid ejection). This is because the intensity of the electric field near the apex of the pipette is reduced by the 200 micron dielectric layer for thin glass.

Figure 4(b) and 4(c) show the results of liquid solution delivery system experiments ((1) Fluid) and fabricated nanopatterning ((2) nanofabrication). After the dye molecule solution (rhodamine $6 \mathrm{G}$, excitation at 488 $\mathrm{nm}$ ) is ejected onto the substrate through the $\sim 100 \mathrm{~nm}$ aperture nanopipette, fluid continues to spread on the surface by the electro-osmosis effect from the application of an $E$ field for the $\mathrm{Au}$ electrode coated on top of the surface as shown in Fig. 4(b). From the spreading area and speed, the flow rate through the aperture of the pulled nanopipette can be calculated. We determined that the outward fluid speed from the center along one axis (parallel to surface) and the dispersed area of the ejected dye solution are $151 \pm 22 \mathrm{~nm} / \mathrm{s}$ and $28 \pm 3 \mu^{2}$ at $30 \mathrm{~s}$. From these results, we derive the flow rate through the $100 \mathrm{~nm}$ aperture as $2.55 \pm 0.2$
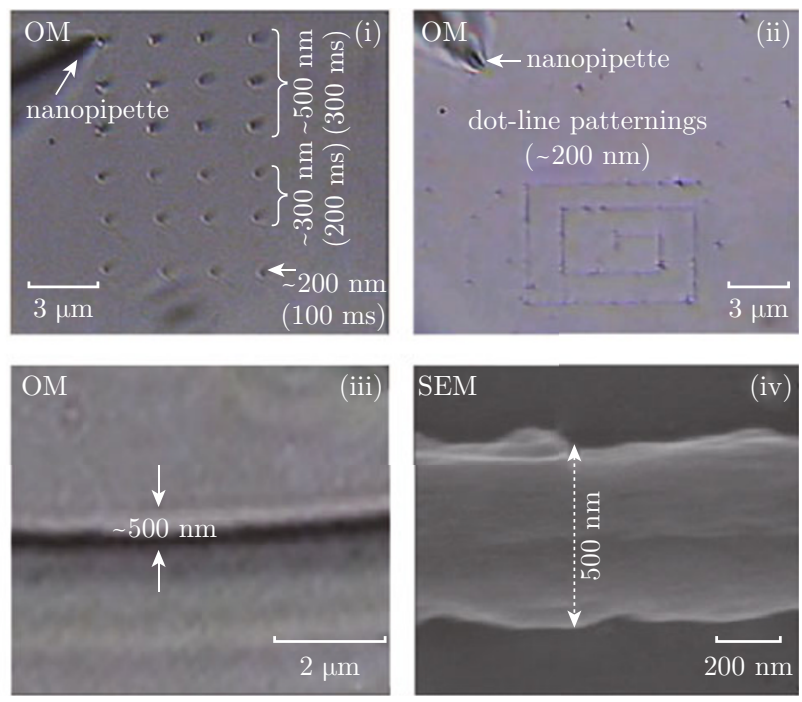

(2) Nanofabrications

(c)

Fig. 4 Fluid and nanofabrication using the proposed system. (a) Schematic for each experiment using fluid and nanofabrication. The bottom electrode is located above (fluid) and under (nanofabrication) the glass substrate; (b) Liquid solution delivery phenomena experiments ((1) Fluid); (c) Fabricated nanopatterns ((2) Nanofabrication). Nanoscale dot (i) and line (ii) patterns. Vertically grown nanowires using a $5 \mathrm{~nm}$ Au nanoparticle solution (iii), (iv). 
femtoliters/s. This rate is approximately calculated by deriving the spread volume using i) the aperture diameter $(100 \mathrm{~nm})$, ii) the assumption of fluid thickness (500 $\mathrm{nm})$ with a hydrophilic substrate, and iii) the evaporation rate $\left(\sim 0.28 \mathrm{mg} / \mathrm{m}^{2} \cdot \mathrm{s}\right)$ of the ejected liquid from the surface. Note that the small variation of the fluid volume (mass) was detected by monitoring the resonance frequency and phase of the QTF over the entire fluid experiment, which does not significantly affect the experiment. Moreover, the distance between the apex of the pipette and the substrate are fixed at the moment of liquid ejection. Fluid phenomena are simply observed by the OM without affecting QTF sensor conditions. Contrary to the fluid experiments, the volume of the ejected liquid can be suppressed by using the $\mathrm{Au}$ electrode coated under the surface, which exerts only an electrostatic force without the electro-osmosis effect with a simple flipping method of the Au-coated substrate. Figure 4(c) shows the result of nanofabrication on a clean $\mathrm{Au}$-coated glass substrate using the $5 \mathrm{~nm}$ Au nanoparticle solution $\left(5 \pm 0.2 \mathrm{~nm}, \mathrm{H}_{2} \mathrm{O}\right.$ buffer, $0.01 \%$ concentration, BB International Co.)-filled nanopipette. After ejection of the liquid solution onto the substrate, the solution was evaporated in air, and nanoparticles formed a nanopatterning. Part (i) of Fig. 4(c) shows the results of nanoscale dot patterning performed by a sudden retraction after liquid ejection. The dot sizes in the pattern depend on the dwell time after the start of liquid ejection. Each dwell time of 100, 200, 300 ms resulted in different dot diameters of $\sim 200,300,500$ $\mathrm{nm}$, respectively. Note that clear observation of surface OM images is due to the $20 \mathrm{~nm}$ thick Au sputtered coating, which is thin enough to avoid obscuring phenomena on the substrate (< skin depth). Part (ii) of Fig. 4(c) shows dot-line patterns produced by lateral movement of the nanopipette tip, while the distance from the surface is sustained with a gap of about $10 \mathrm{~nm}$ with the QTF-AFM system after liquid ejection, performed using a digital feedback system. The set point of the feedback loop is defined by the degradation value of the QTF sensor output amplitude as the tip approaches in the presence of a liquid meniscus forming between the apex of the tip and substrate. Note that the actual position of the tip can fluctuate within $2 \sim 3 \mathrm{~nm}$ at a reference position $10 \mathrm{~nm}$ from the surface. This is because identical conditions of ejected liquid flow cannot be achieved due to randomly located nanoparticle clusters inside the pipette near the apex, which influence on the QTF signals when they flow through the aperture. Discrete patterning (dot-line) may be caused by capillary effects $[30,31]$, which are a significant issue in nanoscale material transferred printing techniques. The capillary force through surface tension for a hydrophilic surface (glass) grips a contact area, and results in the deformation of the line as dot-line shapes through lateral tip movement. This effect may be caused by the discrete positioned clusters of nanoparticles in solu- tion near the apex of the pipette. Part (iii) and (iv) of Fig. 4(c) show OM and SEM images, respectively, of vertically grown nanowires by the continuous slow retraction of the nanopipette. After liquid ejection, the nanoparticles aggregate within the boundary of the pipette aperture and form the shape of a nanowire coincident with the tip retraction. The $\sim 500 \mathrm{~nm}$ diameter of the nanowire is fabricated using a $\sim 500 \mathrm{~nm}$ aperture nanopipette. A nanowire would be suggestive of a continuous solid phase, however the fabricated nanowires show different characteristics compared to bulk properties. For instance, these fabricated nanowires melt with no sustaining the shape as time passes. We have begun to investigate liquid-solid phase coexistence of particle aggregated nanowires under conditions of fast evaporation of water and self-assembly of solvent and nanoparticles, and liquid confinement among the aggregated nanoparticles during the retraction of the pipette tip.

\section{Various materials (nanowire/PDMS)}

Various target nanomaterials such as nanowires and PDMS were used for nanofabrication with the proposed technique. First, a Si nanowire solution (diameter of 50 $\pm 2 \mathrm{~nm}$, length of $2 \pm 0.5 \mu \mathrm{m}, \mathrm{H}_{2} \mathrm{O}$ buffer, $\sim 0.002 \%$ concentration) grown by the VLS method [32] is used for demonstration of the system. Alignment of the nanowires on the desired region, which is a challenging problem in the field of nanowire electronics [33,34], is performed and shown in Fig. 5(a). The low density of the Si nanowire solution filled inside of the $\sim 200$ $\mathrm{nm}$ aperture nanopipette results in the alignment of nanowires upon the approach, ejection, and evaporation of the liquid. Part (i) and (ii) of Fig. 5(a) show the alignment of the straight and bent, respectively, single Si nanowires (diameter of $\sim 50 \mathrm{~nm}$, length of $\sim 2$ $\mu \mathrm{m}$ ) by lateral movement of the piezoelectric element after the nanowire solution is ejected onto the surface. In particular, the on-demand alignment of nanowires is shown in part (iii) and (iv) in Fig. 5(a). The individual alignment of nanowires is difficult to achieve due to the random positions of the nanowires on the substrate during developing nanowire-based electronic devices. For demonstrating on-demand alignment (connection) of nanowires, we first fabricated patterns of two closely separated regions using the relatively high volume liquid ejection and evaporation of deionized water through the $10 \mu \mathrm{m}$ aperture pulled pipette filled with the Si nanowire solution including a supercritical organic solvent (Toluene). After the micro-aperture pipette tip approaches the surface with the application of an $E$ field, the inside solvent is ejected onto two closed regions of the substrate. The solvent residue then forms two separated patterns by evaporation of deionized water within a distance of $\sim 1 \mu \mathrm{m}$. As a result, the two nearly closed regions using a solvent-filled nanopipette 
are connected through the two nanowire-aggregated lines by another $\sim 100 \mathrm{~nm}$ Si nanowire solution-filled nanopipette ( $\sim 300 \mathrm{~nm}$ aperture). The region of interest is monitored by a nanopipette/QTF-AFM system combined with an OM. Moreover, the straight alignment of a $\sim 20 \mathrm{~nm}$ single Si nanowires was achieved and aligned on the desired spot using the $\sim 100 \mathrm{~nm}$ nanopipette.

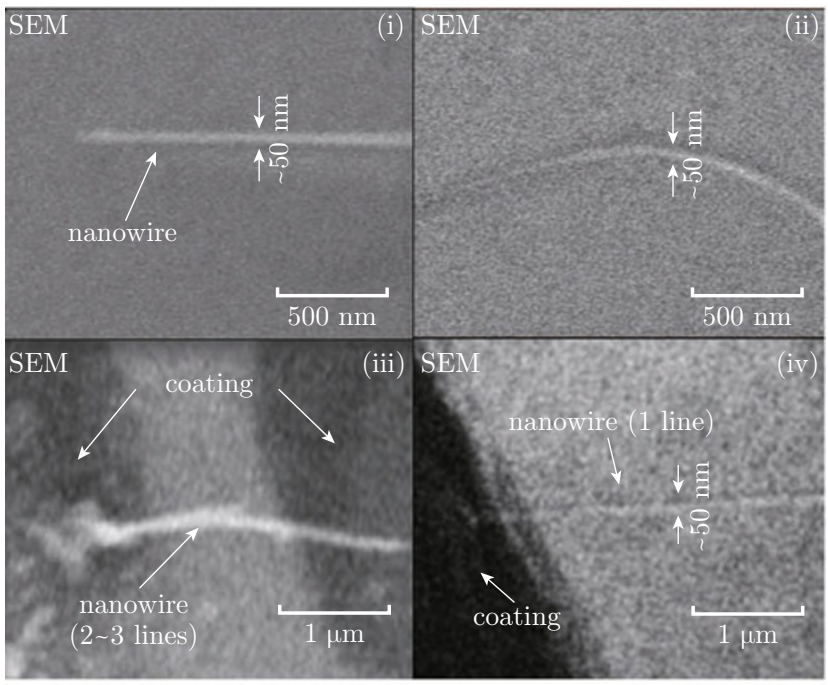

(a)

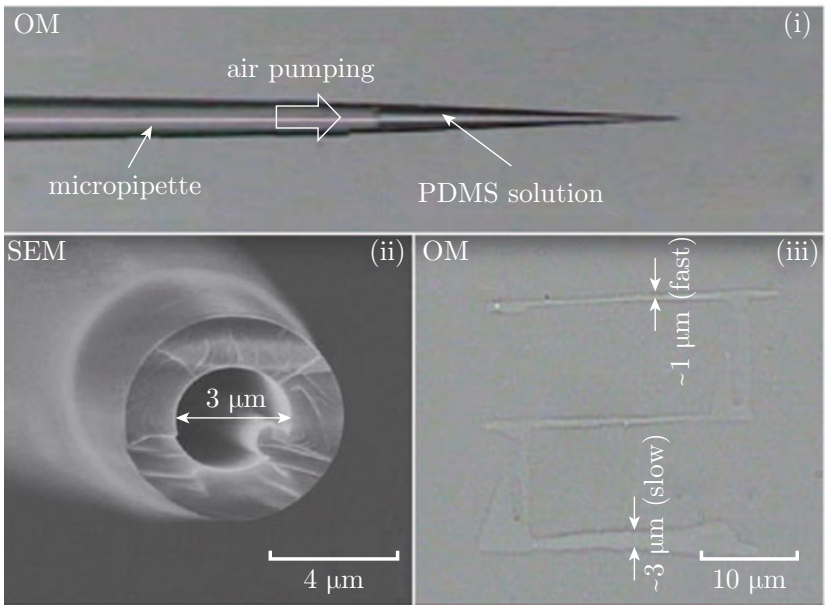

(b)

Fig. 5 Results for various materials. (a) Si nanowire solution, SEM images of the nanoscale aligned (i) straight and (ii) bent nanowires/(iii) 2 - 3 nanowire-aggregated lines and (iv) single line of a Si nanowire, which connects a path between two solvent-coated regions using the Si nanowire solution ( $\sim 50 \mathrm{~nm}$ diameter, $\sim 2 \mu \mathrm{m}$ length) with a positionresolved system; (b) PDMS solution. The PDMS solution can be ejected onto the glass surface and by air pumping with (i) the micro-injector and directly forms a pattern using (ii) a $\sim 3 \mu \mathrm{m}$ aperture pipette. (iii) The line width of the pattern $(1-3 \mu \mathrm{m})$ can be controlled by the pumping flow rate and the writing speed.

PDMS is a versatile material useful in the field of biosystems, such as for soft matter and lab-on-a-chip (LOC) applications [35-37], where microscale patterns are generally fabricated by photolithography or imprinting [38]. In this work, we demonstrate the direct patterning of PDMS, which is mixed in a 1:10 ratio with a curing agent and base (Dow Corning, Sylgard 184 elastromer kit) using the proposed liquid delivery system. An air pumping system with a commercial microinjector (Manual Injector, Sutter Instrument Co.) is used to eject the PDMS solution onto the substrate, rather than an $E$ field, as shown in Fig. 5(b). This method was used because the PDMS solution has a relatively high viscosity comparing to low viscous solutions. Thus, a relatively larger aperture diameter of the pipette is needed to overcome the high surface tension near the apex. The reduction in surface tension with increasing aperture diameter results in solution ejection with no $E$ field. A $3 \mu \mathrm{m}$ aperture pipette was used to fabricate the microscale PDMS pattern. Surface observation was made using the OM. Nanoscale patterning is still challenging due to the relatively high viscosity of the PDMS solution. The line width can be varied by the pumping rate and writing speed. In this experiment, air is pumped by turning a micrometer 10 small division (0.01 inch lateral movement), which yields $\sim 100 \mathrm{~nL}$ with a constant air pressure ( 10 psi), and the writing speed varies from $500 \mathrm{~nm} / \mathrm{s}$ (patterning size of $3 \mu \mathrm{m}$ ) to $2 \mu \mathrm{m} / \mathrm{s}$ (patterning minimum size of 1 $\mu \mathrm{m})$. With this tool, one can easily design and obtain microscale patterning for soft matter or LOC without a complicated fabrication process.

\section{Conclusion}

We showed the direct observation of nanofabrication using an OM combined with a nanopipette/QTF-AFM system. Systematic approaches and fluid/nanofabrication are demonstrated using the proposed system. In addition, solution delivery and nano/micropatterning were performed with nano/microscale fabrication of nanowires and PDMS. In summary, we showed that resolution of the proposed system is $\sim 200 \mathrm{~nm}$ with an $\mathrm{Au}$ nanoparticle solution and $\sim 1 \mathrm{~m}$ with a PDMS solution. We are improving the resolution of the proposed device for smaller apertures and increasing the speed of the tip. One may achieve small and stable nanopatterns with various materials, such as biomolecules, organic/inorganic materials, and carbon nanotubes (CNT) with this technology. We expect that this system can be adjusted and applied to a variety of research fields in bio-nanotechnology.

\section{Acknowledgments}

This work was supported by the National Research Foundation of Korea (NRF) grant funded by the Korea government (MSIP) (2013-056344), Ba- 
sic Science Research Program through the National Research Foundation of Korea (NRF) funded by the Ministry of Education, Science and Technology (2013R1A6A3A03063900), and the Brain Korea 21. Wonho Jhe acknowledges the support from Center for Nano-Liquid, and Corey Stambaugh acknowledges support from the National Science Foundation OISE Grant \#0853104.

\section{References}

[1] D. Cyranoski, "Science education: Reading, writing and nanofabrication", Nature 460, 171-172 (2009). http://dx.doi.org/10.1038/460171a

[2] B. D. Gates, Q. Xu, M. Stewart, D. Ryan, C. G. Willson and G. M. Whitesides, "New Approaches to Nanofabrication: Molding, Printing, and Other Techniques", Chem. Rev. 105(4), 1171-1196 (2005). http://dx.doi.org/10.1021/cr030076o

[3] S. Y. Chou, P. R. Krauss and P. J. Renstrom, "Imprint lithography with 25-nanometer resolution", Science 272(5258), 85-87 (1996). http://dx .doi .org/10. 1126/science. 272.5258.85

[4] D. R. S. Cumming, S. Thoms, S. P. Beaumont and J. M. R. Weaver, "Fabrication of $3 \mathrm{~nm}$ wires using $100 \mathrm{keV}$ electron beam lithography and poly(methyl methacrylate) resist", Appl. Phys. Lett. 68(3), 322-324 (1996). http://dx.doi.org/10.1063/1.116073

[5] M. Remeika and A. Bezryadin, "Sub-10 nanometre fabrication: molecular templating, electron-beam sculpting and crystallization of metallic nanowires", Nanotechnology 16(8), 1172-1176 (2005). http://dx . doi.org/10.1088/0957-4484/16/8/032

[6] R. D. Piner, J. Zhu, F. Xu, S. Hong and C. A. Mirkin, "Dip-pen nanolithography", Science 283(5402), 661663 (1999). http://dx.doi.org/10.1126/science. 283.5402 .661

[7] S. Hong, J. Zhu and C. A. Mirkin, "Multiple ink nanolithography: toward a multiple-pen nano-plotter", Science 286(5439), 523-525 (1999). http://dx.doi.org/ $10.1126 /$ science. 286.5439 .523

[8] M. Hong, J. Bae, K. Kim and W. Jhe, "Scanning nanolithography using a material-filled nanopipette", Appl. Phys. Lett. 77(16), 2604-2606 (2000). http://dx.doi. org/10.1063/1.1319181

[9] A. Bruckbauer, L. Ying, A. M. Rothery, D. Zhou, A. I. Shevchuk, C. Abell, Y. E. Korchev and D. Klenerman, "Writing with DNA and protein using a nanopipet for controlled delivery", J. Am. Chem. Soc. 124(30), 88108811 (2002). http://dx.doi.org/10.1021/ja026816c

[10] F. Iwata, S. Nagami, Y. Sumiya and A. Sasaki, "Nanometre-scale deposition of colloidal Au particles using electrophoresis in a nanopipette probe", Nanotechnology 18(10), 105301 (2007). http://dx.doi. org/10.1088/0957-4484/18/10/105301

[11] A. Meister, M. Liley, J. Brugger, R. Pugin and H. Heinzelmann, "Nanodispenser for attoliter volume deposition using atomic force microscopy probes modified by focused-ion-beam milling", Appl. Phys. Lett. 85(25), 6260-6262 (2004). http://dx.doi.org/ $10.1063 / 1.1842352$

[12] S. Deladi, N. R. Tas, J. W. Berenschot, G. J. M. Krijnen, M. J. de Boer, J. H. de Boer, M. Peter and M. C. Elwenspoek, "Micromachined fountain pen for atomic force microscope-based nanopatterning", Appl. Phys. Lett. 85(22), 5361-5363 (2004). http://dx.doi.org/ 10.1063/1.1823040

[13] A. Fang, E. Dujardin and T. Ondarcuhu, "Control of droplet size in liquid nanodispensing", Nano Lett. 6(10), 2368-2374 (2006). http://dx.doi.org/ 10.1021/nl061694y

[14] B. M. Kim, T. Murray and H. H. Bau, "The fabrication of integrated carbon pipes with sub micron diameters", Nanotechnology 16(8), 1317-1320 (2005). http://dx. doi.org/10.1088/0957-4484/16/8/056

[15] M. Schrlau and H. H. Bau, "Carbon-based Nanoprobes for cell biology", Micro Nano Fluid $7(4)$, 439-450 (2009). http://dx.doi.org/10.1007/ s10404-009-0458-x

[16] J. A. Thompson and H. H. Bau, "Microfluidic beadbased assay: theory and experiments", J. Chrom. B. 878(2), 228-236 (2010). http://dx.doi.org/10.1016/ j.jchromb. 2009.08.050

[17] J. Thompson, X. Du, J. M. Grogan, M. Schrlau and H. H. Bau, "Polymeric microbead arrays for microfluidic applications", J. Micromech. Microeng. 20(11), 115017 (2010). http://dx.doi.org/10.1088/0960-1317/20/ $11 / 115017$

[18] S. Ito and F. Iwata, "Nanometer-scale deposition of metal plating using a nanopipette probe in liquid condition", Jpn. J. Appl. Phys. 50, 08LB15 (2011). http://dx.doi.org/10.1143/JJAP.50.08LB15

[19] A. Lewis, Y. Kheifetz, E. Shambrodt, A. Radko, C. Sukenik and E. Khatchatryan, "Fountain pen nanochemistry: atomic force control of chrome etching", Appl. Phys. Lett. 75(17), 2689-2691 (1999). http://dx.doi.org/10.1063/1.125120

[20] R. R. Gruter, J. Voros and T. Zambelli, "FluidFM as a lithography tool in liquid: spatially controlled deposition offluorescent nanoparticles", Nanoscale 5(3), 1097 (2013). http://dx.doi.org/10.1039/C2NR33214K

[21] F. J. Giessibl, "Atomic resolution of the silicon (111)-(7x7) surface by atomic force microscopy", Science 267(5194), 68-71 (1995). http://dx . doi . org/10. 1126/science. 267.5194.68

[22] F. J. Giessibl, "Advances in atomic force microscopy", Rev. Mod. Phys. 75(3), 949 (2003). http://dx.doi. org/10.1103/RevModPhys.75.949

[23] M. Lee, B. Sung, N. Hashemi and W. Jhe, "Study of a nanoscale water cluster by atomic force microscopy", Faraday Discuss. 141, 415-421 (2009). http://dx.doi. org/10.1039/B807740C

[24] H. Choe, M.-H. Hong, Y. Seo, K. Lee, G. Kim, Y. Cho, J. Ihm and W. Jhe, "Formation, manipulation, and elasticity measurement of a nanometric column of water molecules", Phys. Rev. Lett. 
95(18), 187801 (2005). http://dx.doi.org/10.1103/ PhysRevLett.95.187801

[25] M. Lee and W. Jhe, "General theory of amplitudemodulation atomic force microscopy", Phys. Rev. Lett. 97(3), 036104 (2006). http://dx.doi.org/10.1103/ PhysRevLett.97.036104

[26] S. An, J. Kim, K. Lee, B. Kim, M. Lee and W. Jhe, "Mechanical properties of the nanoscale molecular cluster of water meniscus by high-precision frequency modulation atomic force spectroscopy", Appl. Phys. Lett. 101(5), 053114 (2012). http://dx.doi.org/10. $1063 / 1.4740083$

[27] M. Lee, J. Jahng, K. Kim and W. Jhe, "Quantitative atomic force measurement with a quartz tuning fork", Appl. Phys. Lett. 91(2), 023117 (2007). http://dx. doi.org/10.1063/1.2756125

[28] S. An, M. Hong, J. Kim, S. Kwon, K. Lee, M. Lee and W. Jhe, "Quartz tuning fork-based frequency modulation atomic force spectroscopy and microscopy with all digital phase-locked loop", Rev. Sci. Instrum. 83(11), 113705 (2012). http://dx.doi.org/10.1063/ 1.4765702

[29] S. An, C. Stambaugh, G. Kim, M. Lee, Y. Kim, $\mathrm{K}$. Lee and W. Jhe, "Low-volume liquid delivery and nanolithography using a nanopipette combined with a quartz tuning fork-atomic force microscope", Nanoscale 4(20), 6493-6500 (2012). http://dx.doi. org/10.1039/C2NR30972F

[30] J. K. Hwang, S. Cho, J. M. Dang, E. B. Kwak, K. Song, J. Moon and M. M. Sung, "Direct nanoprinting by liquid-bridge-mediated nanotransfer moulding", Nat. Nanotech. 5, 742-748 (2010). http://dx.doi.org/10. 1038/nnano. 2010.175

[31] J. Park, M. Hardy, J. Kang, K. Barton, K. Adair, D. K. Mukhopadhyay, C. Lee, M. S. Strano, A. G. Alleyne, J. G. Georgiadis, P. M. Ferreira and J. A. Rogers, "High-resolution electrohydrodynamic jet printing", Nat. Mater. 6, 782-789 (2007). http://dx.doi.org/ $10.1038 /$ nmat 1974
[32] R. S. Wagner and W. C. Ellis, "Vapor-liquid-solid mechanism of single crystal growth", Appl. Phys. Lett. 4(5), 89-90 (1964). http://dx.doi.org/10.1063/1. 1753975

[33] M. Liu, Y. Chen, Q. Guo, R. Li, X. Sun and J. Yang, "Controllable positioning and alignment of silver nanowires by tunable hydrodynamic focusing", Nanotechnology 22(12), 125302 (2011). http://dx.doi. org/10.1088/0957-4484/22/12/125302

[34] A. W. Maijenburg, M. G. Maas, E. J. B. Rodijk, W. Ahmed, E. S. Kooij, E. T. Carlen, D. H. A. Blank and J. E. ten Elshof, "Dielectrophoretic alignment of metal and metal oxide nanowires and nanotubes: a universal set of parameters for bridging pre-patterned microelectrodes", J. Col. Inter. Sci. 355(2), 486-493 (2011). http://dx.doi.org/10.1016/j.jcis.2010.12.011

[35] A. A. S. Bhagat, P. Jothimuthu and I. Papautsky, "Photodefinable polydimethylsiloxane (PDMS) for rapid lab-on-a-chip prototyping", Lab Chip 7(9), 11921197 (2007). http://dx.doi.org/10.1039/B704946C

[36] T. Scharnweber, R. Truckenmller, A. M. Schneider, A. Welle, M. Reinhardt and S. Giselbrecht, "Rapid prototyping of microstructures in polydimethylsiloxane (PDMS) by direct UV-lithography", Lab Chip 11(7), 1368-1371 (2011). http://dx.doi.org/10. 1039/COLC00567C

[37] K. J. Regehr, M. Domenech, J. T. Koepsel, K. C. Carver, S. J. Ellison-Zelski, W. L. Murphy, L. A. Schuler, E. T. Alarid and D. J. Beebe, "Biological implications of polydimethylsiloxane-based microfluidic cell culture", Lab Chip 9(15), 2132-2139 (2009). http://dx.doi.org/10.1039/B903043C

[38] L. Engel, J. Shklovsky, D. Schrieber, S. Krylov and Y. Shacham-Diamand, "Freestanding smooth micron-scale polydimethylsiloxane (PDMS) membranes by thermal imprinting", J. Micromech. Microeng. 22(4), 045003 (2012). http://dx.doi.org/10. $1088 / 0960-1317 / 22 / 4 / 045003$ 